\title{
SOME USES OF THE ALTERNATING CURRENT IN HIGH SCHOOL WORK.
}

\author{
Bx L. M. ParrotT, \\ Saginaw High School.
}

I believe the alternating current may be made of almost universal application in outlining fundamental principles, and as an auxiliary apparatus it is almost indispensable.

In my own work the subject of electricity is opened by attempting to get before the pupil a clear conception of the terms used and the definite meaning of each of these terms. This naturally leads into the subject of the fundamental properties of the current, and this point, to my mind, is the basic principle upon which the entire superstructure is founded. A clear conception of these fundamental properties is therefore absolutely essential, and must be made clear and definite by actual existing evidence.

I believe we will all agree that the greatest and most important, if not the fundamental property is manifested by what is called, for lack of a better term, the field of force; and until the actual relation between the current and its accompanying lines of force is made unquestionably evident to the pupil's mind, all that the subject can give him will be but a hazy and imperfect idea. Experiment only can settle the question, and for this purpose a small current is not satisfactory. We are reduced therefore, in the great majority of schools at least, to the problem of either using a large number of cells, to be made by the teacher at this time, or else making use of the city's incandescent plant, which supplies an alternating current. The latter is cheap, always ready, easily manipulated, and will show many of the essential relations as completely as a direct current.

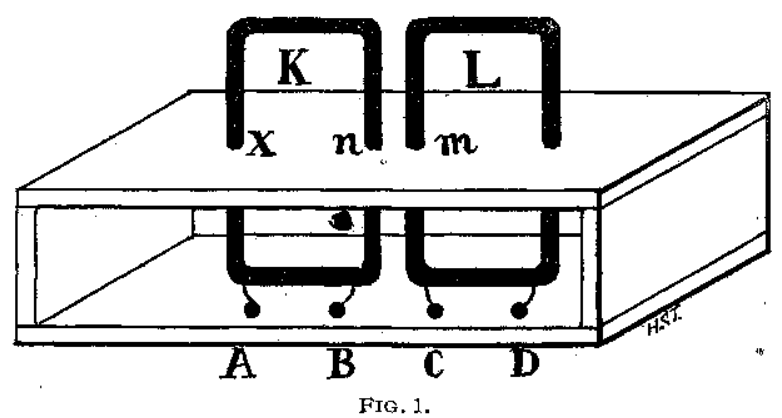


To illustrate: If we connect the terminals of the alternating current, through a suitable rheostat, to the posts $A$ and $B$ (Fig. I) respectively, so that, it surges back and forth through the coil $K$, and sprinkle iron filings around the point $x$ the circular lines of force will be evident, thus showing the existence of a field of stress of some nature encircling the current-carrying wire. These lines of stress, however, may be more than mere circles of force, and are possibly endowed with some kind of a direction property as well, or possess what might be termed a head and tail with the heads all turned one way with reference to the direction of the current. This question may also be clearly demonstrated by means of our alternating current.

Assume, if you will, that this stress about the wire, or so called field of force is endowed with a direction property; then if we assume a particular direction in which the lines are all headed with reference to the direction of the current, for example, as indicated; 4 and now arrange two parallel wires with current flowing in opposite directions, by connecting the terminals at $A$ and $D$, and an extra wire from $B$ to $C$, so that the current flows through both coils represented in Fig. I, the combined field

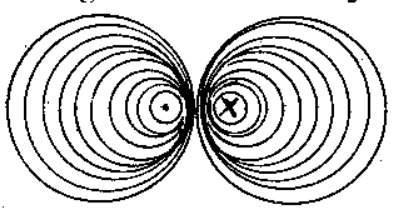
would necessarily be something like this: With a thick and highly strained field between the two wires. Now, still supposing this same direction property, if we exchange the terminals $A$ and $B$, thus reversing the current in one of the coils, we will have it passing through the two central wires, parallel, and in the same direction. $\rightarrow$ Under this condition a line of force circling around the wire $m$ would, when attempting to exert its force, or as some would say, when attempting to pass, between the wires, come under the influence of the current in wire $n$, and thus be prevented from exerling itself in the space between. the wires, and, affected by this influence, must continue on around the wire $n$, and so enclose both wires, presenting a field like this:

Conneating up respectively in the two ways indicated, we see the fields are so arranged.

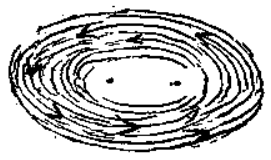
This effect must therefote be due to the cause indicated, and 
we are inevitably compelled to accept the fact that all lines of force do possess a direction property of some nature. That is, when the current goes one direction, the lines of force also have a direction property, dependent on the current direction. Assuming a head and tail for each line, we mean the heads are all turned one direction around the wire. Which direction we call head, or which tail depends only on convention, and convention has established that the arrow head in the ffgure is to be called head, or, as ofttimes falsely stated, the line passes around the wire in the direction indicated, the dot meaning current coming up out of the paper.

Some additional facts may be deduced from the two fields represented (Figs. 2 and 3 ).

Turning our attention to these figures and remembering that these lines are a stress or strain around the current-carrying wire, and should therefore act like stretched elastic bands, we note certain actions likely to result. In Fig. 2 these lines are crowded and need more room. Therefore, by their very nature, they should press these wires apart.

On the other hand, the situation as exemplified in Fig. 3 should be such as to cause the wires to be drawn together.

These facts may be illustrated by means of two coils set up as indicated, the alternating current being fully as effective in proving these well known laws as a direct one.

This simple apparatus may be briefly described as follows: $A$ and $B$ are two coils in series, $A$ stationary, and $B$ supported by a central axis $m n$ upon which it may freely turn, its ends dipping into the respective grooves, which are filled with mercury.

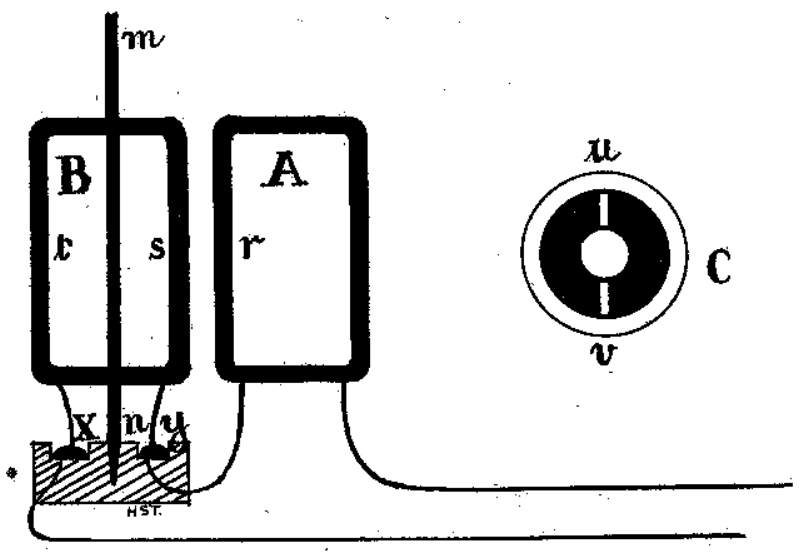

FIG. 4. 
These grooves are illustrated by the top view $C$ and are so arranged that when the coils are parallel with each other, current is flowing in opposite directions at $s$ and $r$ respectively. $B$ is rotated because of the repulsion set up by the crowded condition of the lines of force, and $t$ is attracted. $t$ would thus stop near $\mathrm{r}$, but just as it reaches that point, the ends $x$ and $y$ pass over the bridges $u$ and $v$ (built of paraffine), reversing the current in the coil $B$, thus insuring continual rotation. The terminals $x$ and $y$ are double ones, each being made up of two terminals in parallel spread apart slightly more than the width of the bridges $u$ and $v$. This will cut out the coil $B$ for an instant each half revolution doing no harm thereby, and will prevent the self-induction spark caused by breaking the circuit from fusing the terminals. This experiment not only illustrates these fundamental laws, but may, at the same time, give the pupil a starting point from which to work out the motor and its commutator.

One other fact may be shown from the conditions above. When the current passes the same direction in two parallel wires, we have seen that the field of force encircles both the wires, and that iron seems to be an excellent conductor or retainer of this stress. If therefore, we wind a wire in the form of a solenoid we have an extended condition of the same idea, the combined result of which is that we have lines of force running through the whole coil and returning round the outside.
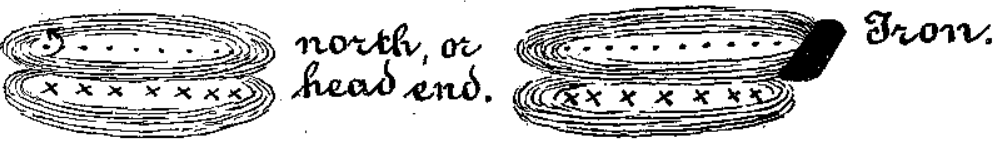

If now a piece of iron were placed near the end of such a group of parallel lines of force, these lines, like stretched elastic bands, in attempting to manifest their own nature, would pull the iron closer, and if not too large, within the coil. This illustrates so called magnetic attraction, which is nothing more nor less than the lines of force attempting to manifest their own nature. This fact may be illustrated by hanging such a coil by one end, and then holding a large nail near the lower end. It will be lifted out of the hand. A perceptible heating may be felt manifested in the nail which is practically absent when the same experiment is tried with a direct current. This will certainly aid in giving some idea of what takes place within the nail at each alternation of the current, which, as we have previously seen, is accom- 
panied by a reversal in the direction of the lines of force sent through the coil and hence through the nail. The vibrations caused by these reversals will also be plainly perceptible to the hand holding it. Magnetism, to my mind, may be made clearer and more real if it were made to follow and not to precede, as in most texts, the experimental evidence of this fundamental property of a current: its field of force.

By some such simple experiments as these, all with "home made" apparatus, I believe nearly all the fundamental properties of the electric current may be easily demonstrated with the city's incandescent or alternating system. It is cheap and very convenient, and a current of some kind is a practical necessity in every school in order that our young people may obtain a clear idea of the subject. As an auxiliary aid in the lecture room, I find it almost indispensable.

Take, for example, one illustration, the arc light. I confess I cannot well get along without it. With it we may accurately illustrate the law of inverse squares, focus and conjugate foci of concave mirrors, laws of reflection of mirrors, not to mention the value of the stereopticon. How to use it to adequately show the laws of reflection of mirrors may need a little further explanation.

We have a tank about five feet long and four broad with a glass bottom. I support this at its ends and place the light and a mirror (either a large concave or plane) beneath in such a way as to reflect the light upward. Placing water in the tank to the depth of about one-half inch, and a piece of wood, held down with a weight, cut, say, in the form of a concave mirror; by touching the water with the end of a ruler at some point as $\mathrm{x}$, representing the position of the object, the waves will proceed to the mirror and be reflected, coming to a point which represents the position of the image. To send parallel rays to the mirror it is only necessary to touch the whole length of the ruler simultaneously. With the electric light below the tank the whole picture is thus magnified and projected upon the ceiling and the whole problem made evident. 